\title{
Solar antineutrino bounds and magnetic field: spin flavour precession after KamLAND
}

\author{
Joao Pulido*† \\ CFIF-IST Lisboa \\ E-mail: pulido@cfif ist.utl.pt!
}

\begin{abstract}
We use the recent KamLAND observations to predict the solar antineutrino spectrum at some confidence limits. We find a scaling of the antineutrino probability with respect to the magnetic field profile -in the sense that the same probability function can be reproduced by any profile with a suitable peak field value- which can be utilised to obtain a general shape of the solar antineutrino spectrum. This scaling and the upper bound on the solar antineutrino event rate, that can be derived from the data, lead to: 1) an upper bound on the solar antineutrino flux, 2) the prediction of their energy spectrum. We get $\phi_{\bar{\nu}}<4.9 \times 10^{-3} \phi\left({ }^{8} B\right)$ or $\phi_{\bar{\nu}}<1.3 \times 10^{-3} \phi\left({ }^{8} B\right)$ at $95 \% \mathrm{CL}$, assuming Gaussian or Poissonian statistics, respectively. For $90 \%$ CL these become $\phi_{\bar{\nu}}<4.4 \times 10^{-3} \phi\left({ }^{8} B\right)$ and $\phi_{\bar{\nu}}<1.0 \times 10^{-3} \phi\left({ }^{8} B\right)$. This provides an improvement by a factor of 3-10 with respect to existing bounds. These limits are quite general and independent of the detailed structure of the magnetic field in the solar interior.
\end{abstract}

The recent KamLAND experiment [1] searching for antineutrinos of $E_{\bar{\nu}}>1.8 \mathrm{MeV}$ via

$$
\overline{\nu_{e}}+p \rightarrow n+e^{+}
$$

has provided strong indications through the observation of reactor antineutrinos that the solution to the solar neutrino problem is dominated by matter oscillations with a large mixing angle (LMA). Before the appearance of KamLAND data, the resonant spin flavour precession (RSFP) [2-4] with Majorana neutrinos based on a transition magnetic moment, provided very good fits to the solar neutrino data [5]. After adding the KamLAND result, it has become clear that SFP solutions, both resonant and non-resonant, may only be accepted at $99.86 \%$ C.L. or $99.88 \%$ C.L. respectively [6]. Hence SFP cannot be the leading solar neutrino problem solution. It can however be present at a subdominant level. Such a

\footnotetext{
${ }^{*}$ Speaker.

${ }^{\dagger}$ Work done in collaboration with Bhag C. Chauhan (CFIF-IST) and E. Torrente-Lujan (Dep. Fisica, Grupo de Fisica Teorica, Univ. Murcia).
} 
possibility was recently investigated [7], its signature being the appearance of antineutrinos from the Sun $[3,8,9]$. The production of these antineutrinos must predominantly occur as a vacuum oscillation from the Sun to the Earth [7]

$$
\nu_{e_{L}} \rightarrow \bar{\nu}_{\mu_{R}} \rightarrow \bar{\nu}_{e_{R}}
$$

with the first step in this sequence (SFP) taking place inside the Sun.

The amplitude for the $\nu_{e_{L}} \rightarrow \bar{\nu}_{\mu_{R}}$ transition was evaluated in perturbation theory for small $\mu B$, the product of the neutrino magnetic moment by the solar magnetic field and found to be, assuming the LMA solution to be dominant [7]

$$
A\left(\nu_{e_{L}} \rightarrow \bar{\nu}_{\mu_{R}}\right)=\frac{\mu B\left(r_{i}\right) \sin ^{2} \tilde{\theta}\left(r_{i}, E\right)}{g_{2}^{\prime}\left(r_{i}\right)}
$$

In this expression $r_{i}$ denotes the neutrino production point, $g_{2}^{\prime}$ is a function of the interaction potentials $V_{e}, V_{\mu}$ and $\tilde{\theta}\left(r_{i}, E\right)$ is the matter mixing angle. Recently we proposed a more realistic case [10] in which we convolute the neutrino production distribution spectrum with the solar magnetic field profile. In this way, the overall antineutrino production probability is obtained as

$$
P\left(\nu_{e_{L}} \rightarrow \bar{\nu}_{e_{R}}\right)=\frac{1}{2} \sin ^{2} 2 \theta \int\left|A\left(\nu_{e_{L}} \rightarrow \bar{\nu}_{\mu_{R}}\right)\right|^{2} f_{B}\left(r_{i}\right) d r_{i}
$$

where the factor $1 / 2$ comes from averaging over the vacuum oscillations, $\theta$ is the vacuum mixing angle [7], $f_{B}$ represents the neutrino production distribution function for Boron neutrinos [11] and the integral extends over the whole production region.

The essential point is that the ${ }^{8} B$ production spectrum is sharply peaked around 0.054 of the solar radius so this probability is almost independent of the shape of the solar field profile. This can be made clear if one considers the limiting case of an infinitely peaked ${ }^{8} B$ production spectrum

$$
P\left(\nu_{e} \rightarrow \bar{\nu}_{e_{R}}\right)=\frac{\mu^{2}}{2} \sin ^{2} 2 \theta \int \frac{B^{2}\left(r_{i}\right) \sin ^{4} \tilde{\theta}\left(r_{i}, E\right)}{g_{2}^{\prime 2}\left(r_{i}, E\right)} \delta\left(0.054-r_{i}\right) d r_{i}
$$

Hence

$$
P\left(\nu_{e} \rightarrow \bar{\nu}_{e_{R}}\right)=\frac{\mu^{2} \sin ^{2} 2 \theta B^{2}(0.054) \sin ^{4} \tilde{\theta}(E, 0.054)}{2 g_{2}^{\prime 2}(E)} .
$$

This probability $P_{\bar{\nu}}(E)$ is just a function of the neutrino energy $E$ and the dependence on the field is through the value $B(0.054)$ only. This argument would be wrong for $p p$ neutrinos, since their production spread is much larger. So there is an approximate scaling factor for the solar antineutrino production probability $P_{\bar{\nu}}(E)[10]$.

$$
P_{\bar{\nu}}[B(r), E]=k(E) P_{\bar{\nu}}^{0}(E),
$$

where $P_{\bar{\nu}}^{0}(E)$ is the antineutrino production probability corresponding to an arbitrary reference field profile. This scaling factor can be evaluated from (3) and (4) which yields 


$$
k(E)=\frac{\int\left(\frac{B\left(r_{i}\right) \sin ^{2} \tilde{\theta}\left(r_{i}, E\right)}{g_{2}^{\prime}\left(r_{i}, E\right)}\right)^{2} f_{B}\left(r_{i}\right) d r_{i}}{\int\left(\frac{B^{0}\left(r_{i}\right) \sin ^{2} \tilde{\theta}\left(r_{i}, E\right)}{g_{2}^{\prime}\left(r_{i}, E\right)}\right)^{2} f_{B}\left(r_{i}\right) d r_{i}}
$$

The effect of the scaling factor $k$ can be seen in fig.1: different solar field profiles lead to approximately the same antineutrino probability $P_{\bar{\nu}}^{0}(E)$ provided in each case a convenient choice of peak field $B_{0}$ is made and the magnitudes of $B_{0}$ required may be quite different for different profiles. In this way there are profiles more efficient than others for $\bar{\nu}$ production. This efficiency is balanced by the field intensity $B_{0}$ in the vicinity of the neutrino production peak. In fig. 2 we provide a comparison between the ${ }^{8} B$ neutrino production distribution and the possible magnetic field profile. Hence it is seen that profile 3 (dashed line) is the most efficient of the three.

As a consequence of the profile independence of the antineutrino probability shape, it is possible to extract a general solar antineutrino spectrum from the recent KamLAND observations. The normalization of the spectrum can, of course, only be obtained in future from the possible solar antineutrino event rate as seen by KamLAND.

The positron event rate in the KamLAND experiment originated from solar antineutrinos can be written as

$$
S=Q_{0} \int_{E_{e}^{0}}^{E_{e}^{\max }} d E_{e} \int_{E_{m}}^{E_{M}} \epsilon\left(E_{e}^{\prime}\right) R\left(E_{e}, E_{e}^{\prime}\right) \phi_{\bar{\nu}}(E) \sigma(E) d E
$$

Here $Q_{0}$ is a normalization factor which takes into account the number of atoms of the detector and its live time exposure [1] and $E$ is the antineutrino energy, related to the physical positron energy by $E_{e}^{\prime}=E-\left(m_{N}-m_{P}\right)$ to zero order in $1 / M$, the nucleon mass. We thus have $E_{m}=1.804 \mathrm{MeV}$. The parameter $E_{e}$ is the measured positron energy with a lower cut at $E_{e}^{0}=2.6 \mathrm{MeV}$, as set by the KamLAND collaboration, in order to eliminate the background posed by the geo-neutrinos. The functions $\epsilon(\simeq 80 \%)$ and $R$ denote the detector efficiency and the Gaussian energy resolution function of the detector. The antineutrino cross section $\sigma(E)$ was taken from ref.[12]. We considered energy bins of size $E_{e}=0.425 \mathrm{MeV}$ in the KamLAND observation range $(2.6-8.125) \mathrm{MeV}$ [1].

The almost uniqueness of the solar antineutrino spectrum can now be seen. First we note that the spectral flux of solar antineutrinos can be written as

$$
\phi_{\bar{\nu}}(E)=\Phi_{\bar{\nu}} f(E)
$$

where $\Phi_{\bar{\nu}}$ denotes the total solar antineutrino flux and the function $f(E)$ is normalized to one. Alternatively one can obviously write

$$
\phi_{\bar{\nu}}(E)=P_{\bar{\nu}}(E) \phi_{B}(E)
$$

so the scaling behaviour that applies to $P(E)$ also applies to the spectral flux $\phi_{\bar{\nu}}(E)$, in other words the energy shape of $\phi_{\bar{\nu}}(E)$ is almost independent of the field profile as can be 
seen in fig.3. Here the three curves, corresponding to the same profiles as in fig.2, were obtained for quite different values of the peak field.

Finally we derive an upper bound on the solar antineutrino flux from the KamLAND experiment $\Phi_{\bar{\nu}}$. We apply separately Gaussian probabilistic considerations to the global rate in the whole energy range, $E_{e}=(2.6-8.125) \mathrm{MeV}$, and Poissonian considerations to the event content in the highest energy bins $\left(E_{e}>6 \mathrm{MeV}\right)$ where KamLAND observes no signal. We take

$$
S_{\bar{\nu}}^{\text {sun }}=S_{\text {obs }}-S_{\text {react }}(L M A)
$$

where $S_{o b s}=54.3 \pm 7.5$ and $S_{\text {react }}(L M A)$ is the signal expected for the best fit parameters of $\operatorname{KamLAND}\left(\Delta m_{21}^{2}=6.9 \times 10^{-5} \mathrm{eV}^{2}, \sin ^{2} 2 \theta=1\right), S_{\text {react }}=49 \pm 1.3$. At $90(95) \% \mathrm{CLs}$ '

$$
S_{\text {obs }}-S_{\text {react }}=k S_{\bar{\nu}}^{0}<17.8(20.2)
$$

For the Gaussian case we equate for each arbitrary solar field profile the event rate $\mathrm{S}$ to 17.8 (20.2) events and extract the corresponding antineutrino flux

$$
\Phi_{\bar{\nu}}=\int_{E_{m}}^{E_{M}} \phi_{\bar{\nu}}(E) d E
$$

generating that number of events. We obtain

$$
\Phi_{\bar{\nu}}<0.0044 \Phi_{B} \quad(90 \% C L)
$$

and

$$
\Phi_{\bar{\nu}}<0.0049 \Phi_{B} \quad(95 \% C L) .
$$

For the five highest energy bins $E_{e}=6-8.125 \mathrm{MeV}$ no positrons from reactor antineutrinos are expected. Here KamLAND observes no signal. We translate this negative result into an upper bound on the solar antineutrino flux, the one that can generate events in this range. We use Poisson statistics. Confidence intervals are $\left[0, \epsilon_{C L}\right]$ where $\epsilon_{C L}$ denotes the maximum allowed number of events at each particular CL: $\epsilon_{C L}=2.44$ for 90\%CL and 3.09 for $95 \%$ CL. We therefore get

$$
\begin{gathered}
\Phi_{\bar{\nu}}<0.0010 \Phi_{B} \quad(90 \% C L) \\
\Phi_{\bar{\nu}}<0.0013 \Phi_{B} \quad(95 \% C L) .
\end{gathered}
$$

These results are 3 to 10 times stricter than previously existing bounds from LSD [14].

Finally, the near independence of the antineutrino probability leads to an antineutrino spectrum which is a modification of the parent ${ }^{8} B$ one in a profile independent way. A comparison of the two spectra is displayed in fig. 4 which clearly shows the peak shift and distortion introduced by the antineutrino probability. 
To summarize, now that SFP is ruled out as a dominant effect for the solar neutrino deficit, it is important to investigate its still remaining possible signature in the solar neutrino signal, namely an observable $\bar{\nu}_{e}$ flux. From the antineutrino production model expound here, an upper bound on the solar antineutrino flux can be derived, namely $\phi_{\bar{\nu}}<$ $4.9 \times 10^{-3} \phi\left({ }^{8} B\right)$ and $\phi_{\bar{\nu}}<1.3 \times 10^{-3} \phi\left({ }^{8} B\right)$ at $95 \% \mathrm{CL}$, assuming respectively Gaussian or Poissonian statistics. For $90 \% \mathrm{CL}$ we found $\phi_{\bar{\nu}}<4.4 \times 10^{-3} \phi\left({ }^{8} B\right)$ and $\phi_{\bar{\nu}}<1.0 \times 10^{-3} \phi\left({ }^{8} B\right)$ which shows an improvement relative to previously existing bounds from LSD [14] by a factor of 3-10. These are practically independent of the detailed magnetic field profile in the core and radiative zone and the energy spectrum of this flux is also found to be nearly profile independent. Furthermore, in view of the relation $\phi_{\bar{\nu}}(E)=\phi_{B}(E) P_{\bar{\nu}}(E)$, the same effect also leads to a profile independent antineutrino spectrum.

Note Added

At the time of preparation of the present manuscript we learned that the KamLAND Collaboration has presented new data [15] corresponding to 0.28 kton-year (251 days) and derived a new upper bound on solar antineutrinos of $2.8 \times 10^{-4}$ of the standard solar model ${ }^{8} B$ flux. This bound improves ours by a factor $3-4$.

The work of BCC was supported by Fundação para a Ciência e a Tecnologia through the grant SFRH/BPD/5719/2001. E.T-L acknowledges many useful conversations with P. Aliani, M. Picariello and V. Antonelli, the hospitality of the CFIF (Lisboa) and the financial support of the Spanish CYCIT funding agency.

\section{References}

[1] K. Eguchi et al. (KamLAND Collaboration), Phys. Rev. Lett. 90, 021802 (2003).

[2] J. Schechter and J. W. F. Valle, Phys. Rev. D 24, 1883 (1981); ibid. 25, 283 (1981) Erratum.

[3] C. S. Lim and W. J. Marciano, Phys. Rev. D 37, 1368 (1988).

[4] E. Kh. Akhmedov, Sov. J. Nucl. Phys. 48, 382 (1988), Yad. Fiz. 48 (1988) 599 (in Russian); Phys. Lett. B 213, 64 (1988).

[5] B. C. Chauhan and J. Pulido, Phys. Rev. D 66, 053006 (2002).

[6] J. Barranco, O. G. Miranda, T. I. Rashba, V. B. Semikoz and J. W. F. Valle, Phys. Rev. D 66 (2002) 093009 [arXiv:hep-ph/0207326].

[7] E. K. Akhmedov and J. Pulido, Phys. Lett. B 553, 7 (2003).

[8] E. Kh. Akhmedov, Sov. J. JETP 68690 (1989), Zh. Eksp. Teor. Fiz. 95 (1989) 1195 (in Russian); R. S. Raghavan, et al., Phys. Rev. D 44, 3786 (1991); C. S. Lim et al., Phys. Lett. B 243, 389 (1990); E. Kh. Akhmedov et al., Phys. Rev. D 48, 2167 (1993).

[9] E. Torrente-Lujan, JHEP 04, 054 (2003); Phys. Lett. B 441, 305 (1998); ibid. B 494, 255 (2000); Nucl. Phys. Proc. Suppl. 87, 504 (2000); P. Aliani et al., JHEP 02,025 (2003).

[10] B. C. Chauhan et al., Phys. Rev. D68 (2003) 033015.

[11] See J. N. Bahcall's homepage, http://www.sns.ias.edu/ jnb/.

[12] P. Vogel and J. F. Beacom, Phys. Rev. D 60, 053003 (1999).

[13] K. Hagiwara et al. (Particle Data Group Collaboration), Phys. Rev. D 66, 010001 (2002). 
[14] M. Aglietta et al., Pisma Zh. Eksp. Teor. Fiz. 63, 753 (1996) [JETP Lett. 63, 791 (1996)].

[15] [KamLAND Collaboration], arXiv:hep-ex/0310047. 

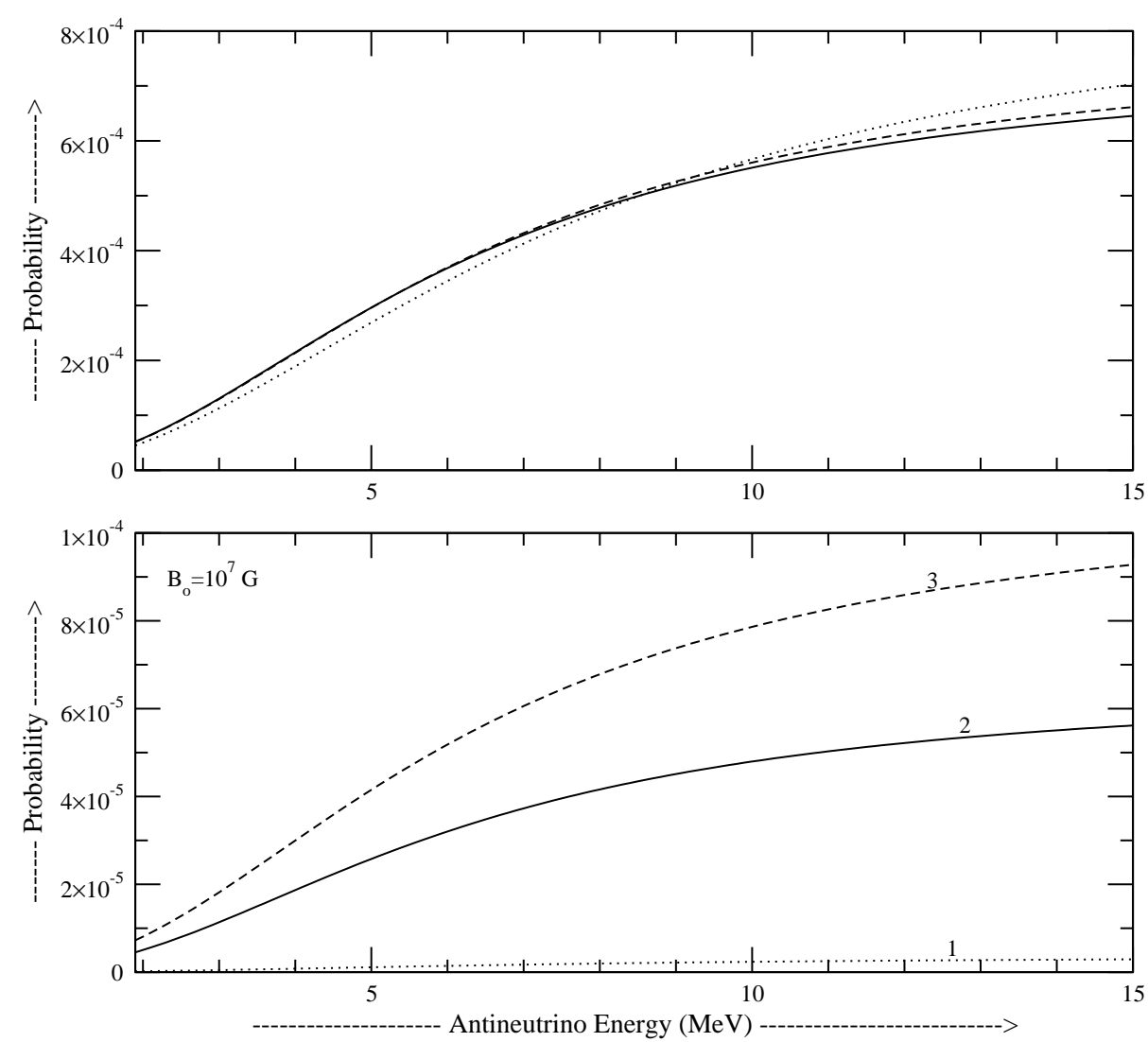

Figure 1: Antineutrino probabilities for three different solar field profiles (see also fig.2). Upper panel: the peak field is chosen in each case so as to produce the same event rate in KamLAND. Lower panel: the same value of the peak field $\left(B_{0}=10^{7} G\right)$ is seen in each case to lead to probabilities of quite different magnitudes. 

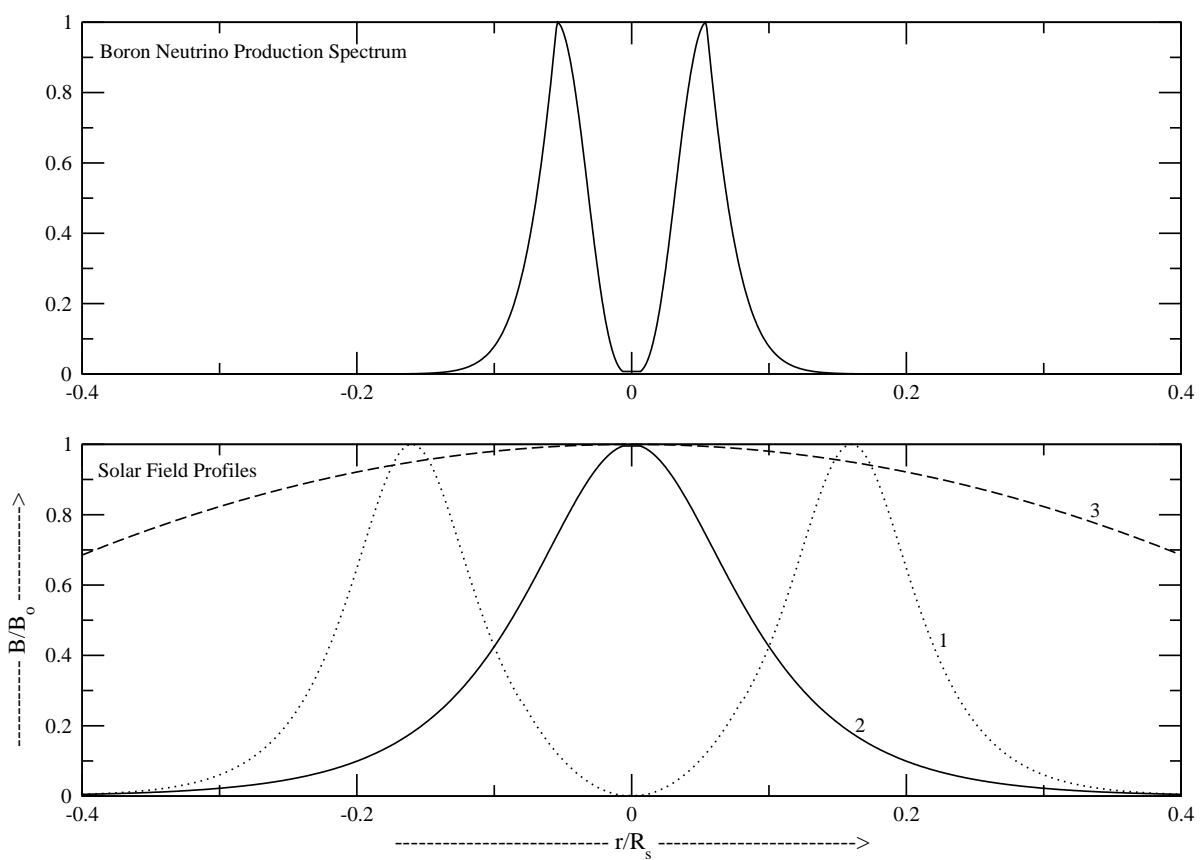

Figure 2: Upper panel: ${ }^{8} B$ neutrino production spectrum (in arbitrary units) as a function of the radial coordinate. Lower panel: the three solar field profiles considered in the main text normalized to $B_{0}$, the peak field value.

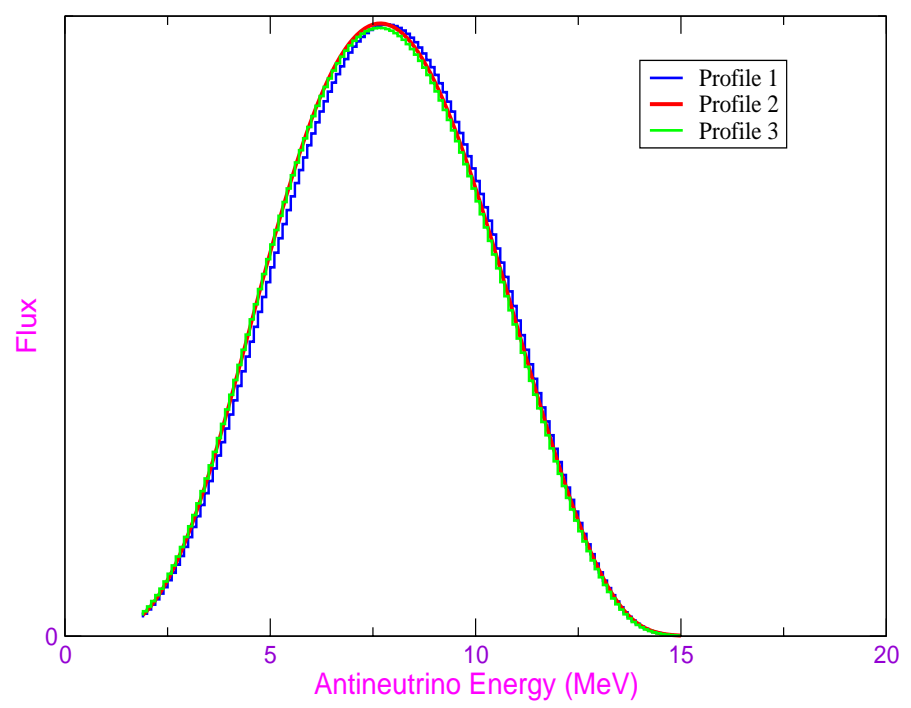

Figure 3: Solar antineutrino spectra for three different solar field profiles. 


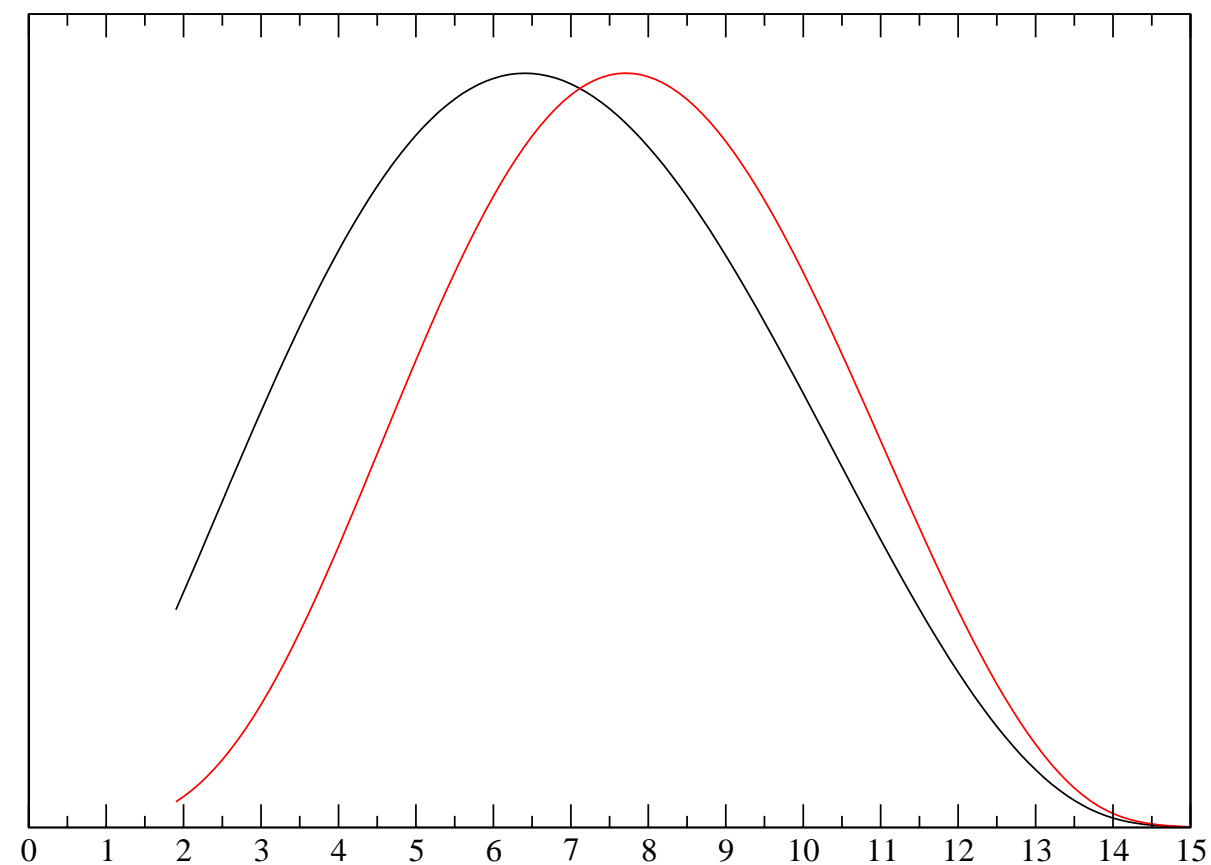

Figure 4: The expected solar antineutrino spectrum (red) and the ${ }^{8} B$ neutrino one (black) [11], both normalized to unity, showing the peak shift and the distortion introduced by the antineutrino probability. Units are in $\mathrm{MeV}$. 